\title{
EXPOSICIÓN LABORAL A HONGOS EN UNA PLANTA DE PROCESAMIENTO DE CAFÉ
}

\author{
ROSA MARÍA ALONSO ESPADALÉ, XAVIER SOLANS LAMPURLANÉS, \\ ANGELINA CONSTANS AUBERT \\ Centro Nacional de Condiciones de Trabajo. \\ Instituto Nacional de Seguridad e Higiene en el Trabajo. Barcelona.
}

\section{RESUMEN}

El café verde, en función del tratamiento que recibe después de la cosecha, puede llegar a las plantas de procesamiento contaminado por hongos, pudiendo producir efectos sobre la salud de los trabajadores que manipulan café, principalmente en forma de reacciones alérgicas y enfermedades respiratorias.

Objetivos: El objetivo de este estudio es determinar la concentración ambiental de hongos a los que están expuestos los trabajadores en las diferentes operaciones que se realizan en una planta de procesamiento de café.

Material y métodos: Las muestras ambientales se obtuvieron por el método de impactación en placa con el equipo M Air T de Millipore. Se determinó la concentración de hongos totales y se identificaron los géneros fúngicos mayoritarios.

Resultados: Se ha obtenido una concentración fúngica ambiental superior a $12000 \mathrm{ufc} / \mathrm{m}^{3}$ durante las operaciones de descarga del café. Los géneros mayoritarios identificados han sido Aspergillus (A. flavus, A. niger y A. ochraceus), Fusarium, Moniliella, Mucor, Rhizopus, Scopulariopsis y Syncephalastrum racemosum.

Conclusión: Los trabajadores de las plantas de procesamiento de café pueden estar expuestos a elevadas concentraciones ambientales de hongos durante las operaciones de descarga del café. Además, debido a la presencia de especies fúngicas toxigénicas, no se puede descartar una potencial exposición a las micotoxinas producidas por estos hongos.

\section{PALABRAS CLAVES}

Procesamiento de café, exposición laboral, hongos ambientales.

\section{ABSTRACT}

The green coffee, depending on the treatment that receives after the crop can reach the processing plants contaminated by fungi. This exposure may produce some effects on the workers health, causing allergic reactions and respiratory diseases.

Objectives: The aim of this study was to determinate the fungi concentrations which are exposed to the workers in the different operations that are done in a coffee processing plant.

Materials and methods: The environmental samples were obtained by plate impactation with the M Air T Millipore sampler. There was determined total fungi concentration and predominant fungi were identified.

Results: Airbone fungal concentrations higher than $12000 \mathrm{ufc} / \mathrm{m}^{3}$ have been found over coffee beans unloading operations. The fungi genera identified were Aspergillus (A. flavus, A. niger y $A$. ochraceus), Fusarium, Moniliella, Mucor, Rhizopus, Scopulariopsis and Syncephalastrum racemosum.

Conclusions: Workers at coffee processing plants may be exposed to high environmental fungi concentrations during coffee beans unloading operations. Furthermore, due to the presence of toxigenic fungi species, a potential exposure to the mycotoxins produced by these fungi may not be dismissed.

\section{KEY WORDS}

Coffee processing, occupational exposure, environmental fungi. 


\section{INTRODUCCIÓN}

El café, en términos de actividad comercial, es la segunda materia prima más importante después del petróleo. La producción mundial se estima en unos 7 millones de toneladas de café verde (sin tostar) anuales y emplea más de 20 millones de personas en todo el mundo. Por lo tanto, se trata de un sector que mueve una gran cantidad de materia prima y que representa una fracción importante de la población laboral.

Generalmente se cultiva en condiciones de calor, lluvia y humedad propios de los países tropicales, pero se procesa en países de clima templado. Esto significa que experimenta marcados cambios, tanto de temperatura como de humedad, desde su recolección hasta que llega a las plantas de procesamiento. Estas condiciones, junto a largos períodos de almacenamiento, pueden favorecer el crecimiento de hongos conocidos como "hongos de almacén", entre los que destacan los géneros Aspergillus y Penicillium. Además, también pueden aparecer otro tipo de hongos, que se desarrollan antes de la cosecha en el campo y que persisten sobre los productos almacenados, denominados "hongos de campo", como los pertenecientes al género Fusarium ${ }^{(1)}$.

Por lo tanto, el café puede llegar a las plantas procesadoras contaminado por hongos y los trabajadores que desarrollan su actividad laboral en estas plantas presentan un riesgo de exposición a estos agentes, que pueden ser liberados al ambiente en forma de bioaerosol (suspensión de partículas en aire compuestas o derivadas de organismos vivos).

Diversos trabajos han relacionado la exposición ambiental a agentes biológicos en plantas de procesamiento de cereales y otros productos de cultivo industrial con efectos sobre la salud de los trabajadores; en plantas de procesamiento de patatas se ha observado que la exposición ambiental a concentraciones elevadas de microorganismos puede producir reacciones alérgicas y/o inmunotóxicas y enfermedades respiratorias en sus trabajadores ${ }^{(2,3,4,5,6,7,8)}$ y en plantas de procesamiento de café reacciones alérgicas y asma laboral ${ }^{(9,10)}$. Además, estudios epidemiológicos realizados para valorar la función respiratoria en trabajadores de estas plantas muestran que la exposición al polvo de café (verde y tostado) puede producir cambios en la función pulmonar y que la prevalencia de síntomas respiratorios crónicos es significativamente más elevada en los trabajadores que manipulan café que en un grupo control ${ }^{t}$. Estos efectos sobre la función respiratoria se pueden deber a la acción vasoconstrictora sobre los bronquiolos que ejerce el polvo tanto del café verde como tostado.

El objetivo de este estudio es determinar la concentración ambiental de hongos en una planta de procesamiento de café e identificar las especies responsables de esta contaminación.

\section{MATERIAL Y MÉTODOS}

\section{Planta de procesamiento de café}

La determinación ambiental se ha realizado en una planta de procesado de café en la que se recibe café verde procedente de diferentes países. El café puede llegar de dos formas: a granel, en camiones tipo Bulk container, o en sacos de $60 \mathrm{~kg}$. La descarga de los camiones Bulk container se inicia con el rasgado manual del saco, realizado por un operario, y la descarga total del café del contenedor a un silo subterráneo.

En cambio, cuando el café viene en sacos de 60 $\mathrm{kg}$ en primer lugar se ha de proceder a la descarga manual de los sacos por parte de un trabajador, que se encarga de sacarlos de la caja del camión y depositarlos en una cinta transportadora que los introduce en la nave de procesamiento, donde posteriormente se vaciará su contenido.

El resto de operaciones se realizan en una nave cerrada, con ventilación natural y sistemas de extracción localizada.

\section{Muestras ambientales}

Las muestras ambientales se obtuvieron por el método de impactación en placa, utilizando el analizador de aire $\mathrm{M}$ Air $\mathrm{T}$ (Millipore), durante las siguientes operaciones 1) descarga de los camiones tipo Bulk container, 2) en la cabina de control del proceso de descarga de estos camiones, 3 ) descarga manual de sacos del interior del camión a la cinta transportadora, 4) vaciado de sacos tipo big-bag y 5) envasado de café verde descafeinado en sacos tipo big-bag.

Asimismo, también se obtuvo una muestra ambiental del exterior de la planta, como referencia de la contaminación fúngica ambiental de la zona.

Como medio de cultivo se empleó el Agar de Extracto de Malta (MEA) (Difco). El volumen de aire captado en las distintas operaciones analizadas varió entre 50 y 250 litros. 
Las placas de MEA se incubaron a $25{ }^{\circ} \mathrm{C}$ durante 3-5 días. Tras el período de incubación se realizó el recuento de las colonias obtenidas. El resultado de la concentración ambiental se expresa como unidades formadoras de colonias por metro cúbico $\left(\mathrm{ufc} / \mathrm{m}^{3}\right)$.

Los distintos géneros fúngicos obtenidos se identificaron por observación macroscópica de las diferentes colonias, aislamiento selectivo en agar Sabouraud Dextrosa (SD) (Difco) e identificación por observación al microscopio óptico de las formas reproductoras, basados en las metodologías de Barnet et al. ${ }^{(14)}$, Campbell et al. ${ }^{(15)}$ y Samson et $a l .^{(16)}$.

\section{RESULTADOS}

La concentración ambiental de hongos en la planta de procesado de café ha variado entre 220 y más de $12000 \mathrm{ufc} / \mathrm{m}^{3}$ (tabla 1).

La concentración más elevada se obtiene durante las operaciones de descarga del café, tanto de los camiones tipo Bulk container como en la descarga manual de los sacos del interior del camión, con valores superiores a $12000 \mathrm{ufc} / \mathrm{m}^{3}$. En el resto de operaciones, la concentración de hongos ha variado entre 220 y $492 \mathrm{ufc} / \mathrm{m}^{3}$, similar a la obtenida en la muestra de referencia $\left(632 \mathrm{ufc} / \mathrm{m}^{3}\right)$.

Tabla I. Concentración ambiental (unidades formadoras de colonias por metro cúbico $\left(u f c / m^{3}\right)$ ) de hongos en distintas operaciones de una planta de procesamiento de café.

\begin{tabular}{|l|c|}
\hline \multicolumn{1}{|c|}{ Operaciones } & Concentración ambiental de hongos \\
\hline $\begin{array}{l}\text { Vaciado de camiones de café tipo Bulk Container (país de } \\
\text { origen Brasil) }\end{array}$ & Incontables $^{(1)}$ \\
\hline $\begin{array}{l}\text { Vaciado de camiones de café tipo Bulk Container (país de } \\
\text { origen Indonesia) }\end{array}$ & Incontables $^{(1)}$ \\
\hline Cabina control de descarga d e camiones & $220 \mathrm{ufc} / \mathrm{m}^{3}$ \\
\hline Descarga manual de sacos de los camiones & Incontables $^{(1)}$ \\
\hline Vaciado de sacos tipo big -bag & $312 \mathrm{ufc} / \mathrm{m}^{3}$ \\
\hline Envasado de café verde descafeinado en sacos tipo big -bag & $492 \mathrm{ufc} / \mathrm{m}^{3}$ \\
\hline Exterior de la planta (referencia) & $632 \mathrm{ufc} / \mathrm{m}^{3}$ \\
\hline
\end{tabular}

(1) $>12000 \mathrm{ufc} / \mathrm{m}^{3}$

La identificación de los hongos (tabla 2) muestra diferencias entre las especies determinadas en el interior de la planta y las obtenidas en la muestra de referencia. Los géneros mayoritarios identificados durante estas operaciones corresponden a Aspergillus, Fusarium, Moniliella, Mucor, Rhizopus, Scopulariopsis y Syncephalastrum.

\section{DISCUSIÓN}

Los resultados obtenidos en este estudio muestran que los trabajadores de una planta de procesamiento de café pueden estar expuestos a elevadas concentraciones ambientales de hongos. La concentración más elevada obtenida, más de $1,2 \times 10^{4}$ $\mathrm{ufc} / \mathrm{m}^{3}$, es 19 veces superior a la hallada en la mues- tra de referencia obtenida en el exterior de la planta, y se alcanza durante las operaciones de descarga del café, independientemente de la forma como se realiza la descarga (café a granel o en sacos) y de su país de origen. Esta contaminación fúngica es similar a la obtenida por Thomas et al. ${ }^{(17)}$ en una planta de procesamiento de café, donde la concentración media de hongos en las distintas operaciones osciló entre $10^{3}-10^{4} \mathrm{ufc} / \mathrm{m}^{3}$, y en la que la concentración más elevada también se alcanzó durante las operaciones de descarga.

Concentraciones similares se han obtenido también en plantas de procesamiento de patatas ${ }^{(3,7)}$, con valores entre 500 y $1,45 \times 10^{4} \mathrm{ufc} / \mathrm{m}^{3}$, hasta $10^{5} \mathrm{ufc} / \mathrm{m}^{3}$ en instalaciones donde se procesan plan- 
Tabla II. Hongos mayoritarios identificados en el interior de la planta de procesamiento de café y en el exterior de la misma (referencia).

\begin{tabular}{|l|c|c|}
\hline \multicolumn{1}{|c|}{ HONGOS IDENTIFICADOS } & $\begin{array}{c}\text { INTERIOR DE LA } \\
\text { PLANTA }\end{array}$ & $\begin{array}{c}\text { EXTERIOR } \\
\text { (referencia) }\end{array}$ \\
\hline Acremonium & + & + \\
\hline Alternaria & + & + \\
\hline Aspergillus flavus & + & - \\
\hline Aspergillus niger & + & - \\
\hline Aspergillus och raceus & + & + \\
\hline Cladosporium & + & - \\
\hline Fusarium & + & - \\
\hline Moniliella & + & - \\
\hline Mucor & + & - \\
\hline Penicillium & + & - \\
\hline Rhizopus & + & - \\
\hline Scopulariopsis & + & + \\
\hline Syncephalastrum racemosum & + & - \\
\hline
\end{tabular}

tas medicinales ${ }^{(18,19)}$, entre $1,7 \times 10^{4} \mathrm{ufc}^{3} \mathrm{~m}^{3}{ }^{(20)} \mathrm{y}$ $8,2 \times 10^{4} \mathrm{ufc} / \mathrm{m}^{3}$ durante el molido de grano de centeno ${ }^{(21)}$ y hasta $1,2 \times 10^{5}$ ufc/m3 obtenidos durante la descarga de soja ${ }^{(22)}$; sin embargo, han sido inferiores a la concentración obtenida durante la manipulación de cereales, donde se han hallado valores superiores a $10^{6} \mathrm{ufc} / \mathrm{m}^{3}(23)$.

En todos estos casos, se ha observado que la contaminación biológica ambiental está muy ligada a la concentración de polvo que se genera durante las distintas operaciones. En este sentido, la determinación de la concentración de hongos en muestras de cereales y en muestras de polvo de cereales mostró contaminación fúngica sólo en el $30 \%$ de las muestras de cereales analizadas, con valores que oscilaron entre 0 y $227,5 \times 10^{3} \mathrm{ufc} / \mathrm{g}$; sin embargo, esta contaminación se observó en todas las muestras de polvo de cereales analizadas, alcanzando concentraciones entre $115 \times 10^{3}$ y $16.700 \times 10^{3}$ ufc/g ${ }^{(24)}$.

Por lo tanto, la elevada contaminación fúngica obtenida durante la descarga del café se puede asociar a la gran cantidad de polvo de café que se genera durante estas operaciones. En este sentido, el cerramiento de la operación de descarga de los camiones Bulk container resultó en una reducción muy significativa de la contaminación ambiental (resultados no mostrados), obteniendo una concentración fúngica ambiental en la zona donde se ubicaba el trabajador de $710 \mathrm{ufc} / \mathrm{m}^{3}$, similar a la hallada en la muestra de referencia. Sin embargo, operaciones como el vaciado de sacos tipo big-bag y el envasado de café en big-bags, en las que también se genera polvo, al disponer de sistemas de extracción localizada, éste no pasa al ambiente y, paralelamente, se evita la contaminación fúngica, obteniendo una concentración ambiental similar a la obtenida en la muestra de referencia.

Los géneros fúngicos mayoritarios en las operaciones de descarga han sido Aspergillus (A. flavus, A. niger y A. ochraceus), Fusarium, Moniliella, Mucor, Rhizopus, Scopulariopsis y Syncephalastrum racemosum; en el caso de Penicillium, Cladosporium y Alternaria se han determinado tanto en el interior como en el exterior de la instalación, no habiendo cuantificado las diferencias en cuanto a su concentración en ambos puntos.

La mayoría de estos hongos son potencialmente alergénicos y la exposición a sus esporas puede alterar las respuestas inmunitarias y la susceptibilidad individual, pudiendo producir enfermedades como rinitis alérgica, asma bronquial y alveolitis alérgica extrínseca ${ }^{(2,19,20)}$. Una flora fúngica similar se ha identificado también en plantas de procesamiento de 
café, donde Aspergillus (A. niger, A. tamarii, A. ochraceus y A. glaucus grupo), Cladosporium, Penicillium, Wallemia sebi y Eurotium spp fueron los géneros ambientales mayoritarios ${ }^{(17)}$ pero también en plantas de procesamiento de patatas, con los géneros Alternaria, Aspergillus (A. fumigatus y A. níger), Candida, Geotrichum, Mucor y Rhizopus, siendo A. niger el hongo mayoritario ${ }^{(3)}$ y durante la descarga y manipulación de cereales, donde Cladosporium, Aspergillus y Penicillium constituyeron el $71-85 \%$ del total de la contaminación fúngica ambiental $^{(22)}$.

Del análisis fúngico de granos de café verde se observó que prácticamente todas las muestras se hallaban contaminadas y, esta contaminación se producía mayoritariamente por Aspergillus (A. ochraceus, A. niger, A. flavus) y, en menor proporción, Cladosporium y Penicillium ${ }^{(25,26)}$.

Entre los distintos hongos que pueden contaminar el café algunos son capaces de producir micotoxinas (toxicogénicos) durante alguna de las etapas de producción, procesado, transporte y almacenamiento. Las micotoxinas son metabolitos secundarios fúngicos capaces de desencadenar distintas alteraciones y cuadros patológicos en el hombre y los animales, poseen actividad carcinógena, teratógena o mútagena y pueden producir desórdenes de tipo hormonal e inmunodeprepresor ${ }^{(27,28)}$.

Entre los hongos identificados en este estudio, se encuentran distintos géneros productores de micotoxinas: Aspergillus, con las especies A. flavus productor de la aflatoxina B1 y A. niger y A. ochraceus productores de la ocratoxina A y Fusarium, productor, entre otras, de las micotoxinas zearalenona, fumonisinas y tricotecenos. La presencia de estos hongos toxigénicos en el ambiente, aunque no implica necesariamente la existencia de micotoxinas, sí puede hacer sospechar su presencia ${ }^{(29)}$; se ha obser- vado que existe una correlación entre la concentración de hongos y la concentración de micotoxinas en polvo de cereales, de forma concreta entre las distintas especies de Fusarium y fusariotoxinas totales ${ }^{(24)}$ y entre Penicillium y ocratoxina $\mathrm{A}^{(30)}$.

Para evitar, o reducir, la contaminación del ambiente por estos agentes biológicos, la medida más efectiva consiste en evitar la dispersión de polvo en el lugar de trabajo. El cerramiento de las operaciones que generen polvo y la instalación de sistemas de extracción localizada permiten reducir de forma significativa la concentración ambiental de estos agentes. Por otro lado, durante las operaciones de descarga, la cabina del camión debe permanecer con las ventanillas cerradas y disponer de un sistema de suministro de aire limpio. Asimismo es muy importante una correcta limpieza de las instalaciones.

El uso de máscaras con filtro P3 o mascarillas autofiltrantes FFP3 puede ser necesario en operaciones como la descarga manual de los sacos de interior de la caja de los camiones.

Otras medidas preventivas generales consisten en una correcta higiene personal y la prohibición de comer o beber en el lugar de trabajo. Finalmente, se debe ofrecer a los trabajadores una correcta información, así como formación, acerca de los riesgos de su puesto de trabajo, los procedimientos de trabajo y las medidas preventivas a adoptar.

En conclusión, los trabajadores de las plantas de procesamiento de café pueden estar expuestos a una elevada concentración ambiental de hongos durante las operaciones de descarga del café. Además, debido a la presencia de especies fúngicas toxigénicas, no se puede descartar una potencial exposición a las micotoxinas producidas por estos hongos. 


\section{BIBLIOGRAFÍA}

1. OIC/FAO/FCPB Directrices para prevenir la formación de moho en el café en: Mejora de la calidad del café mediante la prevención de la formación de moho. Documento ED 1988/06. Organización Internacional del Café. Food and Agriculture Organization of the United Nations. Fondo Común Productos Básicos. FAO, 2006.

2. Douwes J, Thorne P, Pearce N and Heederik D. Bioaerosol health effects and exposure assesment: Progress and prospects. Ann Occup Hyg 2003; 47 (3): 187-200.

3. Dutkiewicz J, Krysinska-Traczyk E, Skorska C, Cholewa G and Sitkowska J. Exposure to airbone microorganisms and endotoxin in a potato processing plant. Ann Agric Environ Med 2002; 9: 225-235.

4. Ewers LM and Tapp LC. Endotoxin exposures during potato processing. Applied Occup and Environ Hyg 2001; 16 (12): 1079-1087.

5. Fung $\mathrm{F}$ and Hughson, WG. Health effects of indoor fungal bioaerosols exposure. Applied Occupational and Environmental Hygiene 2003; 18: 535-544.

6. Hollander A, Heederik D and Kauffman H. Acute respiratory effects in the potato processing industry due to a bioaerosol exposure. Occup Environ Med 1994; 51: 7378.

7. Zock JP, Heederik D, Kromhout, H. Exposure to dust, endotoxin and microorganisms in the potato processing industry. Ann Occup Hyg 1995; 39: 841-854.

8. Zock JP, Heederik D, Doekes G. Evaluation of chronic respiratory effects in the potato processing industry: indications of a healthy worker effect? Occup Environ Med 1998; 55: 823-827.

9. Bernton HS. On occupational sensitization a hazard to the coffee industry. J Am Med Assoc 1973; 223 (10): 1146-1147.

10. Karr RM. Bronchoprovocation studies in coffee worker's asthma. J Allergy Clin Immonul 1979; 64 (6): 650-654.

11. Zuskin E, Valic F and Skuric Z. Respiratory function in coffee workers. Br J Ind Med 1979; 36: 117-122.

12. Zuskin E, Valic F and Kancelljak B. Immunological and respiratory changes in coffee workers. Thorax 1981; 36: 9-13.

13. Zuskin E, Duncan PG and Douglas JS. Pharmacological characterisation of extracts of coffee dusts. Br J Ind Med 1983; 40: 193-198.

14. Barnett HL\& Hunter BB. Illustrated Genera of Imperfect Fungi. 4th edition. Minnesota (USA); APS Press, St. Paul; 1998.
15. Campbell CK, Johnson EM, Philpot CM and Warnock DW. Identification of Pathogenic Fungi. London (UK); Public Health Laboratory Service; 1996.

16. Samson RA, Hoekstra ES, Frisvad JC, et al. Introduction to food and airbone fungi. 6th Edition. Utrecht (The Netherlands); Centraalbureau Voor Schimmelcultures; 2002.

17. Thomas KE, Trigg CJ, Baxter PJ, Topping M, Lacey J, Crook B, Whitehead P, Bennett JB and Davies RJ. Factors relating to the development of respiratory symptoms in coffee proces workers. Br J Ind Med 1991; 48: 314-322.

18. Skórska C, Sitkowska J, Krysinska-Traczyk E, Cholewa G and Dutkiewicz J. (1) Exposure to airbone microorganisms, dust and endotoxin during processing of valerian roots on farms. Ann Agric Environ Med 2005; 12 : 119-126.

19. Skórska C, Sitkowska J, Krysinska-Traczyk E, Cholewa G and Dutkiewicz J. (2) Exposure to airbone microorganisms, dust and endotoxin during processing of peppermint and chamomile herbs on farms. Ann Agric Environ Med 2005; 12: 281-288.

20. Lugauskas A, Krikstaponis A and Sveistyte L. Airbone fungi in industrial environments-Potential agents of respiratory diseases. Ann Agric Environ Med 2004; 11 : 19-25.

21. Lee S-A, Adhikari A, Grinshpun SA, McKay R, Shukla R and Reponen T. Personal exposure to airborne dust and microorganisms in agricultural environments. J Occup Environ Hyg 2006; 3: 118-130.

22. Adhikkari A, Reponen T, Lee S-A and Grinshpun SA. Assessment of human exposure to airborne fungi in agricultural confinements: personal inhalable sampling versus stationary sampling. Ann Agric Environ Med 2004; 11: 269-277.

23. Swan JRM and Crook B. Airborne microorganisms associated with grain handling. Ann Agric Environ Med 1998; 5: 7-15.

24. Krysinska-Tracyk E, Kiecana I, Perkowski J and Dutkiewicz J. Levels of fungi and mycotoxins in samples of grain and grain dust collected on farms in eastern Poland. Ann Agric Environ Med 2001; 8: 269-274.

25. Batista LR, Chalfoun SM, Prado G, Schwan RF and Wheals AE. Toxicogenic fungi associated with processeed (green) coffee beans (Coffea arabica L.). Int J Food Microbiol 2003; 85 (3): 293-300.

26. Martins ML, Martins HM and Gimeno A. Incidence of microflora and ochratoxin A in green coffee beans (Coffea arabica). Food Addit Contam 2003; 20 (12): 1127-1131. 
27. IARC. Monographs on the evaluation of carconogenic risks to humans. Vol 56 Some naturally occurrin substances: food items and constituents, heterocyclic aromatic amines and mycotoxins. Lyon (France). International Agency for Research on Cancer; 1993.

28. IARC. Monographs on the evaluation of carconogenic risks to humans. Vol 82 Some traditional herbal medicines, some mycotoxins, naphthalene and styrene. Lyon (France). International Agency for Research on Cancer; 2002.
29. Desai MR, Ghosh SK. Occupational exposure to airborne fungi among rice mill workers whith special reference to aflatoxin producing A. flavus strains. Ann Agric Environ Med 2003; 10: 159-162.

30. Halstensen AS, Nordby K-C, Elen O and Eduard W. Ochratoxin A in grain dust-Estimated exposure and relations to agricultural practices in grain production. Ann Agric Environ Med 2004; 11: 245-254. 